\title{
New tools for online teaching and their impact on student learning
}

\author{
Carme Huguet, Jill Pearse, Jorge Esteve
}

Department of Geosciences, Universidad de los Andes, Colombia.

\begin{abstract}
In the context of the global Covid-19 crisis, a practical introductory Geosciences course was redesigned to aid student learning in a 100\% virtual format. New materials were created to i) improve disciplinary language range and concept acquisition; ii) make classes more dynamic; iii) provide tools for self-regulated learning and assessment and iv) maintain student motivation. Usefulness of the new materials was evaluated using a voluntary online survey that was answered by $40 \%$ of the students. Additional information was obtained from the university's student evaluation survey.

All tools were well-rated, but self-assessment quizzes and class presentations had the highest overall scores. Students commented on their usefulness in terms of knowledge acquisition and self-assessment. Perhaps not surprisingly, self-assessment quizzes were the one tool students felt kept them more motivated. These were closely followed by class presentations and short inclass quizzes. Students found the online access to all lesson materials very useful for self-paced learning. According to a majority of students, the in-class quizzes and student participation using the digital the whiteboard made classes more dynamic.

Overall, the new strategies succeeded in improving students' learning and independence, but more work is needed to make classes more dynamic, and especially to improve student motivation. Intrinsic motivation is perhaps the most difficult to improve because in a $100 \%$ virtual course, it is difficult to promote student-student interactions and receive visual feedback from the class. In view of the survey results, we introduce bonus activities in order to improve extrinsic motivation.
\end{abstract}

Keywords: Online teaching; independent learning; intrinsic and extrinsic motivation; self-assessment, self-paced learning. 


\section{Introduction}

Due to the Covid-19 global crisis, many undergraduate programs have had to undergo a drastic shift from in-person classroom teaching to online synchronic lectures (e.g. Baber 2020). In view of the need to redesign the course for online teaching, we created new materials to address several key issues for the learning process, namely language and concept acquisition, dynamization, self-regulated learning and motivation. Not being able to demonstrate concepts using hands-on group activities has made it very challenging to keep the student population motivated. It is difficult to make online classes dynamic because of the lack of direct participation and spontaneous interaction among students (Huguet et al. 2020) in large remote-learning groups. Moreover, the diversity of the student population makes it difficult to tap into intrinsic motivation and address the differing levels of previous knowledge in an integrated way. Other challenges, such as the fact that not all students have consistent access to a computer or stable internet connection, became apparent at the end of the first semester of 2020 during the strict Covid-19 nationwide lockdown, which in Colombia lasted over 6 months. Therefore, the course redesign needed to include asynchronic tools to promote autonomy and allow students to self-regulate their learning.

There are some arguments in favor of online learning, such as flexibility and autonomy of the learning process (e.g. Baber, 2020). However, distance learning, especially in the current lockdown context, can result in students feeling unmotivated and isolated, which lowers their academic performance (Baber, 2020): these issues were identified by UNIANDES in several surveys given to students throughout 2020. Science knowledge building must address both intrinsic and extrinsic motivation, and students are more motivated, and learn better, by interacting with classmates and teachers (e.g. Glyn et al., 2009; Bergmann \& Sams, 2012).

In the online version of the course, we addressed extrinsic motivation by giving short, very frequent activities that represented a small fraction of the course grade, and by reducing the weight of single evaluations such as exams. This was done to lower anxiety (Glyn et al, 2009) and to provide more opportunities for students to receive feedback and encourage a sustained effort throughout the course. Intrinsic motivation was perhaps the most challenging part of the course redesign, since we needed to create opportunities for interaction while in isolation, and emphasize the relevance of the course for non-geoscience majors. Because the course is still $100 \%$ virtual (and will likely continue to be until at least 2022), it was crucial to evaluate the overall impact of the newly created materials. We wanted to see how the redesigned tools had influenced new language and concept acquisition. We also evaluated which materials were considered most useful for students to assess their progress during the course, make the course more dynamic and keep learners motivated. Students were asked to participate in a voluntary online survey, in addition to the institutional student surveys, to help us identify areas of improvement. 


\section{Methods}

In the context of the covid-19 worldwide pandemic, an undergraduate geoscience course was redesigned from an in-person hands-on course to a $100 \%$ online format starting in March 2020. Several new tools were created based on the existing literature, and courses provided by pedagogy and technology experts at UNIANDES as well as online platforms. The surveys were designed to assess the students' perception of the usefulness of the tools and whether they had influenced their motivation.

\subsection{New tools introduced}

Class Presentations: Presentations were redesigned to include in each class a list of contents and a glossary of the relevant geoscience terminology and main concepts. A list of ten questions was given at the end, so students could assess their understanding of key concepts when reviewing the material. Videos and short articles were also provided within each presentation to complement and broaden professors' explanations.

In-class quizzes: Each class included a formative assessment, consisting of multiple choice or true/false questions that were delivered during or at the end of the class by means of online surveys that could be answered anonymously. The correct answers were discussed in class, providing immediate feedback.

Post-class self-assessment quizzes: Each lesson had at least 10 questions online that were available from the day of the class presentation onwards and could be done as many times as required by the student. Those questions were designed to be more challenging than the ones in class, calling for the application of novel concepts to specific cases, and in some cases for a revision of the materials provided in class. Students saw only one question at a time and had to complete the quiz in order to get feedback. Feedback was given in the form of correct answers and the reasons why the other answers were incorrect.

\subsection{Evaluation of tools}

We designed an online survey using QualtricsXM (https://www.qualtrics.com/) to assess the usefulness of each material in addressing our five aims. The survey was delivered at the end of the course, and obtained 144 responses from a pool of 357 students. Since participation in the survey was voluntary, and students are required to complete the general university survey for each course as well, we were pleased that $40 \%$ of the students participated. 


\section{Results and discussion}

\subsection{New language and concept acquisition}

A remarkable decline in the vocabulary of university students has been reported, which is even more pronounced for discipline-specific language (Caratozzolo et al, 2019; Twenge et al., 2019). The acquisition of appropriate scientific-disciplinary language is key for professional development and one of the skills regularly requested by employers (Caratozzolo et al, 2019). Hence, one of our aims was to broaden students' Geoscience lexicon. While we expected the glossary at the end of each class to have a prominent role in discipline-specific language acquisition, students found the tool to be only $74 \%$ effective (Fig. 1a). Instead, the self-assessments and class presentations were rated as $80 \%$ and $85 \%$ effective respectively, in improving geoscience language acquisition (Fig. 1a). We believe this may be because students learn new language and concepts better within a context than when given an explicit vocabulary list to learn. The way in which new vocabulary is delivered is key, it has been shown that the timing (before, during or after) is not as important as the manner in which a content area is delivered (Memory, 1990).

For new concept acquisition, students highlighted the relevance of the self-assessment quizzes (84\%) and class presentations (85\%) (Fig. 1b). We believe that the class presentations serve two complementary functions: the professor explains new concepts, and examples are given in a context that helps student's understanding. Both purposes fall within the first levels of Bloom's taxonomy (Bloom \& Krathwohl, 1956), which are necessary but not sufficient. This was then complemented by the the self-assessment quizzes, which were designed to challenge the students to integrate and apply the newly acquired concepts. The selfassessments therefore target the higher levels of Bloom's pyramid (Bloom \& Krathwohl, 1956), thus providing a full spectrum of learning opportunities for students.

\subsection{Self-assessment of learning}

Students' self-assessment is fundamental for meaningful deep learning, self-regulated learning and metacognitive skills development (Orsmond and Merry, 2013). Especially in the current Covid-19 context, it is important to empower students to be independent and evaluate their own knowledge acquisition (Orsmond and Merry, 2013). The opportunity to evaluate understanding outside of the formal grading context helps reduce students' anxiety when undertaking graded evaluations (e.g. Glyn et al, 2009).

Students rated the self-assessments as $87.8 \%$ effective at tracking their learning (Fig. 1c). Learners especially valued being able to access self-assessment quizzes whenever they needed, repeat them as many times as they wanted, and always obtain feedback on their responses. They said it was very useful to prepare for exams, since it gave them a "safe" exam-like environment to test their conceptual understanding. This tool was purely self- 
regulated and independent, so one downside was that teachers did not have access to information on student performance, and could not take any action on that front.

In-class questions and quizzes for which instant feedback was provided were also highly valued (73\%; Fig. 1c) in terms of self-assessment. Since they were completed within the class, students could see if they needed to go over some concepts again, or proceed directly to the more challenging post-class self-assessment quizzes. Some students also viewed these short quizzes as a kind of summary of the key concepts seen during class, and that helped them identify possible gaps in their knowledge acquisition. Questions and quizzes in class also gave the teacher the opportunity to track students' understanding of the concepts, correct misconceptions and emphasize key ideas covered in the lesson. In this case, the limitation was that only students who attended the classes synchronically could take full advantage of these tools. However, the questions and answers were also recorded, so some students reported them to be useful when viewing the recorded classes asynchronically.

\subsection{Making online classes more dynamic}

Even though some students commented that online classes were tedious and repetitive, several of the designed tools were useful to improve the flow and class dynamic (Fig. 1d).

In-class quizzes were again rated the highest for making the course more dynamic (74\%) closely followed by the list of questions at the end of the class presentations and the postclass self-assessment quizzes (71\%) (Fig. 1d). To our surprise, some students suggested increasing the number of non-graded quizzes. This was unexpected, since one of the requests from the university was to markedly reduce student load during the Covid-19 crisis.

Virtual classes were rated as $67 \%$ useful for making the course dynamic (Fig. 1d), which is not a bad result considering that in-person classes include hands-on activities and materials that could not be used in the virtual version of the course.

Although the virtual whiteboard was not included in the set of tools we designed, it was mentioned several times in the comments of the university survey of student perceptions. The opportunity to use the virtual whiteboard to answer questions posed by the teacher was perceived to make the online classes more dynamic. We noted that during in-person classes students were often reluctant to participate verbally or using the board, but the online version provided anonymity, which increased students' willingness to participate and take the risk of answering questions. This was an unexpected benefit of the virtual format. 
a. Useful to aquire specialized Language

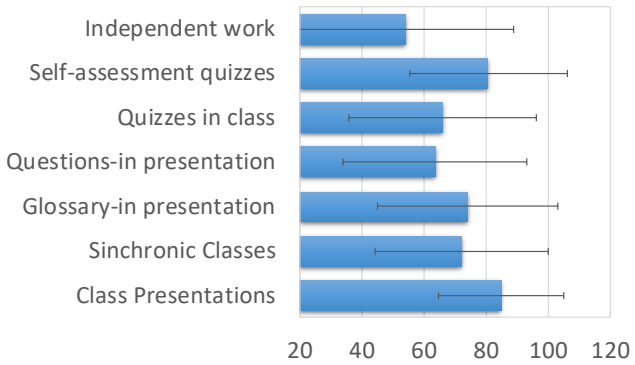

c. Follow my progress in the course

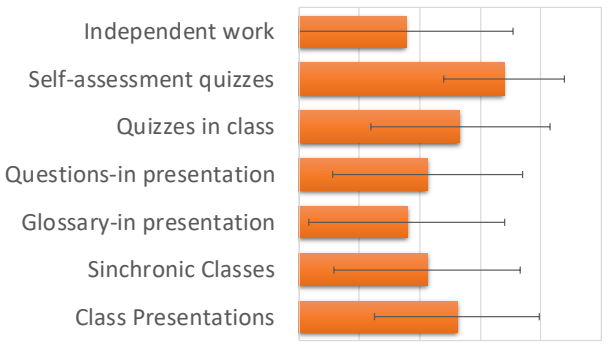

$\begin{array}{llllll}20 & 40 & 60 & 80 & 100 & 120\end{array}$

e. Keep me motivated

Independent work Self-assessment quizzes

Quizzes in class

Questions-in presentation

Glossary-in presentation

Sinchronic Classes

Class Presentations

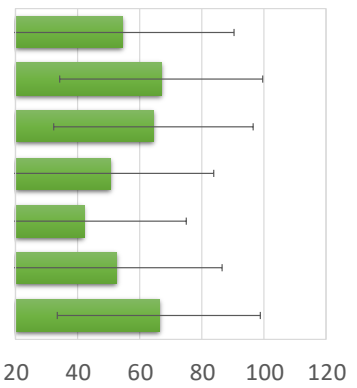

b. Useful to learn new concepts

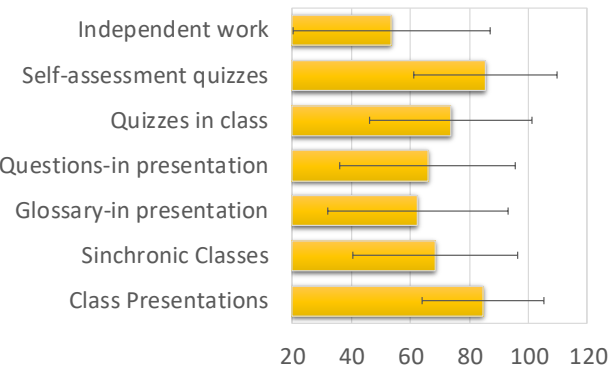

d. Make course more dynamic

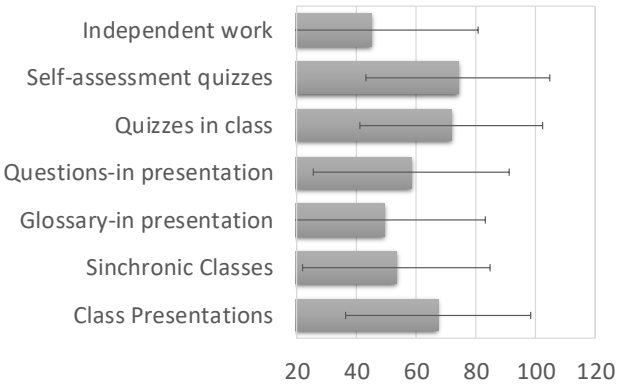

f. Overall tool evaluation

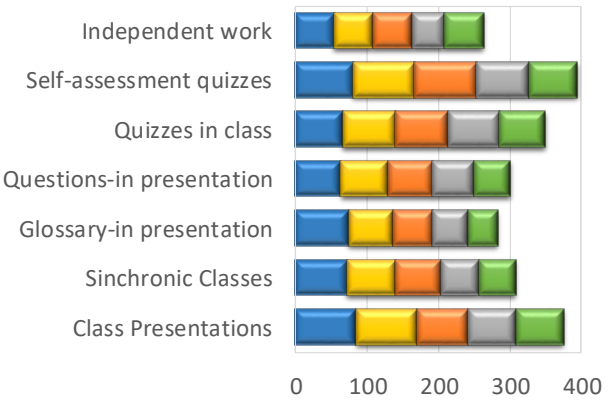

Figure 1. QualtricsXM survey results of tool usefulness on a. lexicon acquisition, $b$. concept acquisition; . selfassessemnt; $d$. dinamization of the course; e. student motivation and f. overall tool ratting. To learn new language (blue), To follow progress (orange), to keep motivated (green), To learn new concepts (yellow) and to make course more dyaic (grey). Data are based on 144 answers and standard deviations are indicated with grey bars 


\subsection{Motivation}

Generally, motivation can be divided into intrinsic and extrinsic (Ryan \& Deci, 2000). Intrinsic motivation happens when the individual considers the information interesting, relevant and enjoyable, whilst extrinsic motivation is related to a specific outcome (such as grades) (Ryan \& Deci, 2000). Science knowledge-building must address both intrinsic and extrinsic motivation to be truly successful (Ryan \& Deci, 2000), and students are more motivated, and learn better, by interacting with classmates and teachers (e.g. Glyn et al. 2009; Bergmann \& Sams, 2012). As educators, we aim to engage students in learning in class in order to motivate them to continue learning on their own (e.g. Van Der Hoeven Kraft et al., 2011). We feel that increasing motivation was the goal we addressed less effectively: students perceived most of the new tools as not particularly effective for increasing motivation (Fig. 1e). Self-assessment quizzes $(66.8 \%)$ and class presentations $(66 \%)$ got the highest evaluation, compared to the other tools (Fig. 1e) but where still in the lowest range.

Class presentations included numerous examples, images and videos that were designed to capture the students' attention. Several students commented on the university survey that being able to access recordings of the classes and review the contents of the presentations motivated them to learn and work on their own. Some also mentioned that having additional videos with in-depth explanations motivated students to learn more on the subject and to explore other sources independently. Thus, it seems intrinsic motivation to learn was fostered by this tool. Learning science for its own sake (intrinsic motivation) is not the general driver in this course since most students will only take it because it is compulsory. Nonetheless, some students commented that they felt the course content was engaging and relevant to their everyday life, which helped keep them motivated. Many of the students are engineers, thus including examples relevant to engineering, and "surprising facts" was found increase intrinsic motivation.

\section{Conclusions}

Overall, the "forced" online version of the course was positively evaluated by the students, with most of the newly-introduced tools considered successful in improving learning. Further work is needed to make online classes more dynamic, interactive, and motivating.

The self-assessment quizzes received the highest overall rating, as they were useful not only for keeping track of progress independently, but also for reducing assessment anxiety, increasing motivation, making classes more dynamic and for learning new vocabulary and concepts.

Class presentations were also very well rated especially for acquiring new knowledge and keeping students engaged. 
In order to address some of these issues we are increasing the number of interactive activities involving the digital whiteboard, as students seem more willing to interact with the teacher and each other in an anonymous virtual setting. We also further reduced the weight of graded individual homework assignments and exams, and included some optional activities that contribute to an overall bonus mark. It has always been impossible to achieve a perfect score (5) in this course, so we hope that a $5 \%$ bonus will contribute to extrinsic motivation.

\section{References}

Baber, H. (2020). Determinants of students' perceived learning outcome and satisfaction in online learning during the pandemic of COVID-19. Journal of Education and e-Learning Research, 7(3), 285-292.

Bergmann, J., \& Sams, A. (2012). Flip your classroom: Reach every student in every class every day. International Society for Technology in Education. www.iste.org/learn/publications/permissions-andreprints.aspx ISBN 978-1-56484-315319

Bloom, B. S., \& Krathwohl, D. R. (1956). Taxonomy of educational objectives: The classification of educational goals, by a committee of college and university examiners. Handbook 1: Cognitive domain. Longmans, New York.

Caratozzolo, P., Alvarez-Delgado, A. \& Hosseini, S. (2019). Strengthening critical thinking in engineering students. International Journal on Interactive Design and Manufacturing, 13, 995-1012.

Glynn, S. M., Taasoobshirazi, G., \& Brickman, P. (2009). Science motivation questionnaire: Construct validation with nonscience majors. Journal of Research in Science Teaching, 46(2), 127-146.

Huguet, C., Pearse, J., Noè, L. F., Valencia, D. M., Ruiz, N. C., Heredia, A. J., \& Avedaño, M. A. P. (2020). Improving the motivation of students in a large introductory geoscience course through active learning. Journal of Geoscience Education, 68(1), 20-32.

Memory, D. M. (1990). Teaching Technical Vocabulary: before, during, or after the Reading Assignment?. Journal of Reading Behavior, 22(1), 39-53.

Orsmond, P., \& Merry, S. (2013). The importance of self-assessment in students' use of tutors' feedback: A qualitative study of high and non-high achieving biology undergraduates. Assessment \& Evaluation in Higher Education, 38(6), 737-753.

Ryan, R. M., \& Deci, E. L. (2000). Intrinsic and extrinsic motivations: Classic definitions and new directions. Contemporary Educational Psychology, 25, 54-67.

Twenge J. M., W. K. Campbell \& R. A. Sherman (2019). Declines in vocabulary among American adults within levels of educational attainment, 1974-2016. Intelligence, 76, 101377,https://doi.org/10.1016/j.intell.2019.101377.

Van Der Hoeven Kraft, K. J., Srogi, L., Husman, J., Semken, S., \& Fuhrman, M. (2011). Engaging students to learn through the affective domain: A new framework for teaching in the geosciences. Journal of Geoscience Education, 59(2), 71-84. 